\title{
Polymyxin B-induced skin hyperpigmentation: a rare case report and literature review
}

\author{
Guanhao Zheng ${ }^{1}$, Li Cao ${ }^{2}$, Zaiqian Che ${ }^{3}$, Enqiang Mao ${ }^{3}$, Erzhen Chen ${ }^{4^{*}}$ and Juan $\mathrm{He}^{5^{*}}$
}

\begin{abstract}
Background: Polymyxin B (PMB), which is regarded as the ultimate antibacterial treatment against some intractable gram-negative bacteria with its outstanding anti-bacterial activities, inflicts several adverse effects on patients. However, skin hyperpigmentaion (SH) induced by PMB is very rare. Here, we report a case of polymyxin B-induced skin hyperpigmentation (PMB-iSH) in a 21-year-old female. To the best of our knowledge, this is the first case of PMB-iSH in China.

Case presentation: A 21-year-old female patient with sepsis received the administration of PMB by intravenous injection for the treatment of multi-drug resistant Klebsiella pneumoniae (MDR-KP) infection. She later suffered from a rare adverse drug reaction (ADR), namely PMB-iSH, after 5-day PMB administration during her treatment. There were multiple red rashes spread on the whole body skin at first. With the rashes fading away, SH with dark round spots appeared, associated with no pain or pruritus. The skin of the head and neck was darkened evidently, and dark brown spots were spread on the skin of trunk and limbs. About a month after her admission, urged by the relatives, the patient was transferred back to the local hospital for further treatment in the end, and her skin color didn't recover to the previous state at that time.
\end{abstract}

Conclusion: Both our case and the literature review highlight that PMB can give rise to SH indeed. Clinicians and pharmacists should attach great importance to this rare pigmentary disorder and further investigation is warranted.

Keywords: Polymyxin B, Skin hyperpigmentation, Sepsis, Case report

\section{Background}

Skin hyperpigmentation (SH) is a common type of skin disease with various inducing factors. Drug-induced skin hyperpigmentation (DiSH) is an important part of $\mathrm{SH}$, accounting for 10 to $20 \%$ of all acquired SH cases [1]. A variety of drugs can trigger $\mathrm{DiSH}$, such as tetracycline, NSAIDs, anti-malarial agents, anti-psychotic agents, cytotoxic agents, amiodarone, etc. [1]. Minocycline is the most common trigger among the aforementioned drugs [2]. Recently, SH has been observed in several patients who received the treatment of intravenous polymyxin $\mathrm{B}$

\footnotetext{
*Correspondence: chenerzhen@hotmail.com; hejuanwin@126.com

${ }^{4}$ Department of Emergency Intensive Care Unit, Ruijin Hospital Affiliated to Shanghai JiaoTong University School of Medicine, EICU 3 Floor, 5 Building, 197 Ruijin No. 2 Road, Huangpu District, Shanghai, China

${ }^{5}$ Department of Pharmacy, Ruijin Hospital Affiliated to Shanghai JiaoTong University School of Medicine, 202 Room, 12 Building, 197 Ruijin No. 2 Road, Huangpu District, Shanghai, China

Full list of author information is available at the end of the article
}

(PMB). Here we discuss a patient with sepsis who was treated by intravenous PMB and suffered from a rare adverse drug reaction (ADR) afterward, namely polymyxin B-induced skin hyperpigmentation (PMB-iSH), on the basis of a literature review of its epidemiology, pathological mechanism, treatment options and prognosis.

\section{Case presentation \\ History and previous admissions}

This was a rare case of PMB-iSH in a 21-year-old female in China. In her postpartum period, the patient suffered from chest pain, fever and even coma for a fortnight. She was sent to the local hospital due to cardiac arrest by 4 times on 27th January 2017. After CPR, she regained consciousness gradually but still was in a continuous febrile state. Klebsiella pneumoniae was isolated from the samples of blood and sputum cultures. Besides, anti-microbial 
therapy hadn't worked effectively since she was treated with cefepime, imipenem and tigecycline.

\section{EICU admission}

The patient was soon admitted to the emergency intensive care unit (EICU) of Ruijin Hospital affiliated to Shanghai Jiao Tong University on 26th April. Upon admissionto our hospital, she was still in fever, unconscious in a dyspneic state, and mechanical ventilation was initiated after tracheotomy with metal tracheal tube.

\section{Examination in EICU \& adjustment for anti-infectious therapy}

A full-body computed tomography (CT) scan identified thickened pericardium, bilateral pleural effusion with multiple exudative focuses, hepatosplenomegaly and pelvic effusion in this patient. Empiric antibiotic treatment was started for Klebsiella pneumoniae infection with piperacillin-tazobactam (4.5 g, intravenously, q.8 h). The sample of microbial sputum culture on 29th April revealed that a large amount of multi-drug resistant Klebsiella pneumoniae (MDR-KP) grew, which was merely susceptible to tigecycline, sulfamethoxazole (SMZ) and $\mathrm{PMB}$. The infection parameters from laboratory examination increased remarkably: hypersensitive $\mathrm{C}$-reactive protein $37.0 \mathrm{mg} / \mathrm{L}[0 \sim 3 \mathrm{mg} / \mathrm{L}]$ and procalcitonin $3.37 \mathrm{ng} / \mathrm{mL}$ $[<0.50 \mathrm{ng} / \mathrm{mL}]$. In light of the above-mentioned examination results, we replaced piperacillin-tazobactam with meropenem ( $2 \mathrm{~g}$, intravenously, q.8 h) and tigecycline (100 mg intravenously q.12 h) with the addition of colistin (1-million unit by aerosol inhalation q.8 h) on 4th May.

\section{Occurrence of PMB-iSH}

After the adjustment for the treatment, her body temperature dropped to normal and remained stable. During this period, repeated cultures of blood, sputum, vaginal secretion and fluid drained from the hip joint were carried out. MDR-KP, susceptible to tigecycline, SMZ and PMB was detected in all the samples. On 18th May, multiple exudations were aggravated in bilateral lungs compared to that upon admission, according to a chest CT scan. As a result, PMB (500,000 units, intravenously, q.12 h) was administered at once. Four days later, there were multiple red rashes spread on the whole body skin. With the rashes fading away, $\mathrm{SH}$ with dark round spots was seen on 23rd May (5 days after intravenous PMB) without pain or pruritus. More dark brown spots were spread on the skin of trunk and limbs, especially on that of lower abdomen, right hand and right foot as depicted in Figs. 1, 2, 3 and 4, although the skin of the head and neck was also darkened evidently. Despite this adverse event, the therapeutic regimen was still applied in consideration of MDR-KP infection of multiple organs. PMB was utilized in the same dosage

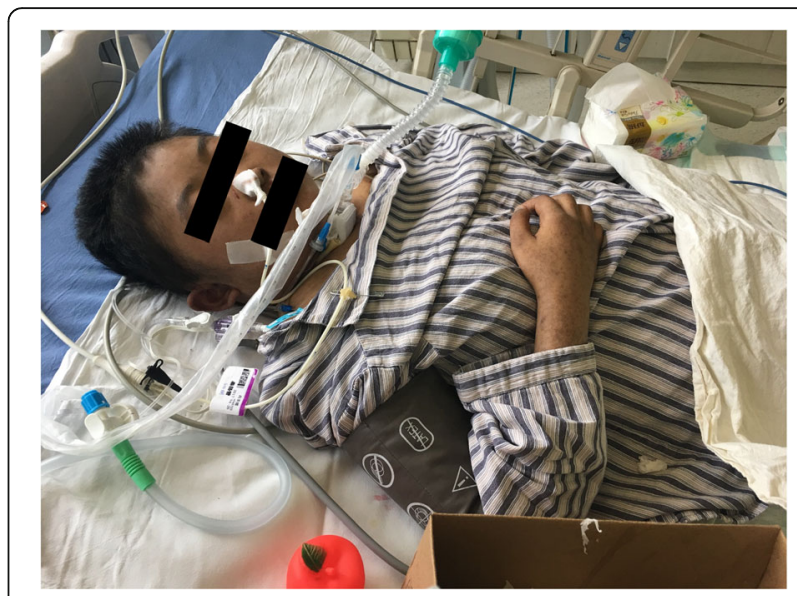

Fig. 1 Hyperpigmentation in the head and face

until the patient was transferred back to the local hospital, and skin biopsy wasn't performed.

\section{Post-treatment course}

On 21st May, she suffered from diarrhea suddenly with intra-abdominal pressure (IAP) increasing to $19 \mathrm{~cm}$ $\mathrm{H}_{2} \mathrm{O}$. About $500 \mathrm{ml}$ black stool was found in the patient' $\mathrm{s}$ excrement. Her serum creatinine was elevated dramatically to $430 \mu \mathrm{mol} / \mathrm{L}$ [53 97 $\mu \mathrm{mol} / \mathrm{L}$ ], and anuria occurred. The laboratory examinations revealed the following results: the level of hemoglobin was significantly reduced to $57 \mathrm{~g} / \mathrm{L}[113 \sim 151 \mathrm{~g} / \mathrm{L}]$ and platelet counts $63 \times 10^{9} / \mathrm{L}\left[101 \sim 320 \times 10^{9} / \mathrm{L}\right]$. Her liver function was impaired moderately: total bilirubin was $43.6 \mu \mathrm{mol} /$ $\mathrm{L}$ [4.7 24 $\mu \mathrm{mol} / \mathrm{L}]$, direct bilirubin was $26.0 \mu \mathrm{mol} / \mathrm{L}$ [0 $6.8 \mu \mathrm{mol} / \mathrm{L}]$, and $\gamma$-GT went up to $103 \mathrm{IU} / \mathrm{L}$ [7 $64 \mathrm{IU} / \mathrm{L}]$. The results of coagulation test were as follows: APTT $50.1 \mathrm{~s}$ [22.3 $38.7 \mathrm{~s}]$, PT $12.9 \mathrm{~s}$ [10.0 $16.0 \mathrm{~s}]$, TT $24.80 \mathrm{~s}$ [14.00 21.00s], Fg $1.5 \mathrm{~g} / \mathrm{L}[1.8 \sim 3.5 \mathrm{~g} / \mathrm{L}]$, FDP $12.5 \mathrm{mg} / \mathrm{L}[0 \sim 5 \mathrm{mg} / \mathrm{L}]$ and D-Dimer $2.66 \mathrm{mg} / \mathrm{L}[<$

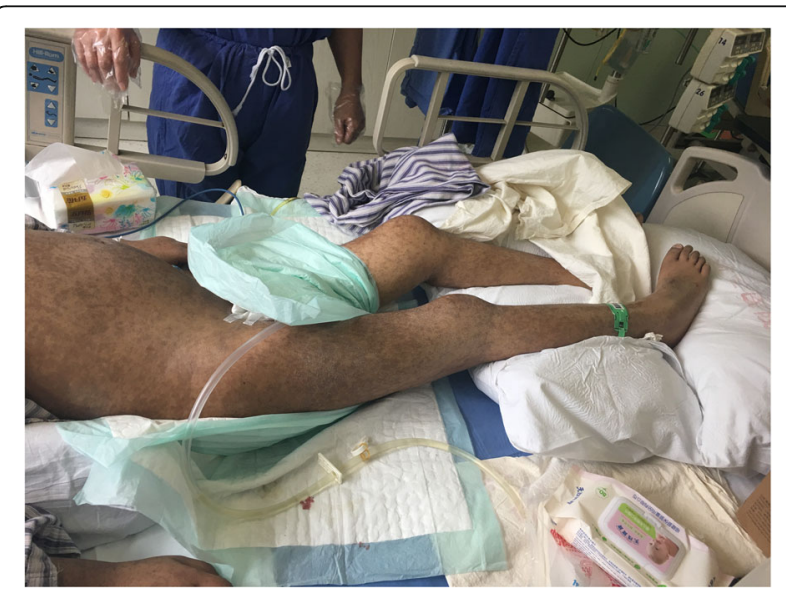

Fig. 2 Hyperpigmentation in the lower abdomen 


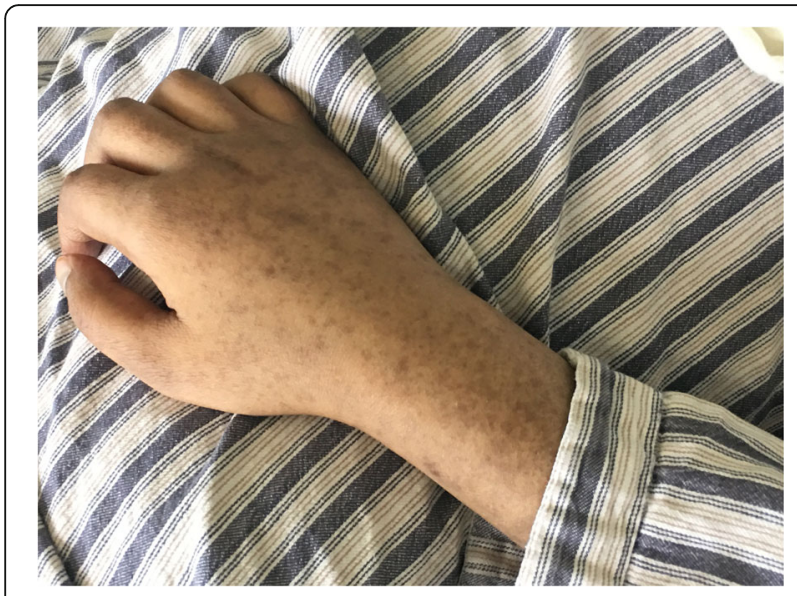

Fig. 3 Hyperpigmentation in the right hand

$0.55 \mathrm{mg} / \mathrm{L}]$. They indicated that the patient suffered from coagulation abnormality and hyperfibrinolysis, hence disseminated intravascular coagulation (DIC). After phlebotomy, the transfusion of cryoprecipitate prepared from plasma frozen within 24 Hours (PF24) started immediately. Meanwhile, low-dose heparin $(3 \mathrm{U} / \mathrm{kg} / \mathrm{h}$ ) was administered prudently for anticoagulation therapy. 2 days later, bleeding disappeared. On 25th May, urged by the relatives, the patient was transferred back to the local hospital for further treatment. At that time, her skin color didn't recover to the previous state. Owing to her relatives' will, we failed to make a follow-up visit. The treatment timeline is shown in Fig. 5.

\section{Discussion \& conclusion}

Of polymyxins and lipopeptide antibiotics, colistin and PMB were isolated from Paenibacillus ploymyxa and has been available in clinical use since 1959. They have excellent anti-microbial activity against a variety of gram-negative bacteria. Unfortunately, the polymyxins

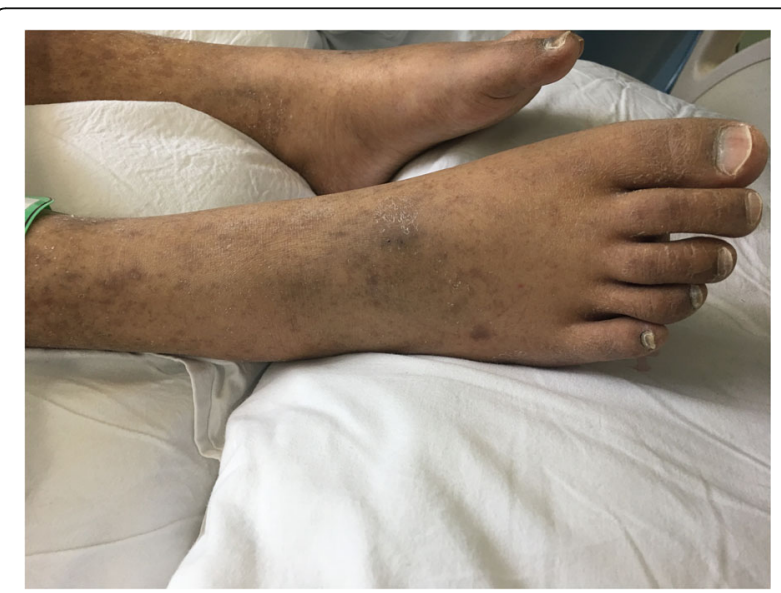

Fig. 4 Hyperpigmentation in the right foot were once replaced by those more effective and safer antibiotics like aminoglycosides on account of their severe nephrotoxicity and neurotoxicity. But in the last few decades, great importance was attached to the polymyxins again by clinicians due to the inevitable appearance of multi-drug resistant (MDR) bacteria. They were even regarded as the ultimate antibacterial treatment against some intractable gram-negative bacteria with their outstanding anti-bacterial activity as well, such as Escherichia coli, Pseudomonas aeruginosa, Acinetobacter baumannii, carbapenem-resistant Enterobacteriaceae, etc. [3-5].

When it comes to the polymyxins, their chemical structure, antimicrobial spectrum and mechanism are extremely similar, although colisitin is a pro-drug for clinical use [4]. The most common ADR of the polymyins is nephrotoxicity, while neurotoxicity and hypersensitivity are also other main ADRs [6]. It is a nonnegligible fact that $\mathrm{SH}$ is proved as a significant side effect induced by $\mathrm{PMB}$, and no $\mathrm{SH}$ cases induced by colistin have been reported.

Only a few case reports on PMB-iSH have been found so far. Knueppel R.C. et al. reported two suspected cases in 2007 for the first time [7]. A patient who suffered from MDR-KP infection was found with $\mathrm{SH}$ after treated by intravenous PMB for 4 days, according to the description by Lahiry S. et al. [8]. Besides, there were several cases in neonates and infants as well. Gothwal S. et al. reported 3 babies who had progressive $\mathrm{SH}$ after intravenous PMB. They maintained that the cumulative effect of PMB might be a possible reason for darkened skin, which was likely to cause the immaturity of kidney functions of neonates, since PMB was excreted through the kidney mainly [9]. Interestingly, Shih L K et al. used Felix von Luschan Skin Color Chart to classify the color of skin tone into 36 different scales. 16 infants were recruited in this study, treated by 15,000 units $/ \mathrm{kg}$ intravenous PMB every $12 \mathrm{~h}$ for 14 days. Based on the average change of skin tone, the skin tone of all the subjects were progressively darkened during the treatment period and reached the peak at the end of the treatment of intravenous PMB (on the 14th day) [10].

In 2016, Mattos K.P. et al. carried out a cohort study on PMB-iSH, which included 60 patients with gram-negative infection who received a 14-day $\mathrm{PMB}$ treatment. PMB-iSH was found in $15 \%$ of the subjects usually on the 3rd day of the treatment period. Their skin color were darkened to various shades of brown, particularly in the neck and face [11]. It was reported in another study that this pigmentary disorder was found in $8 \%$ of 249 patients who used PMB [12].

To the best of our knowledge, the case previously presented is the first case of PMB-iSH in China. On the basis of Naranjo Adverse Drug Reaction Probability 


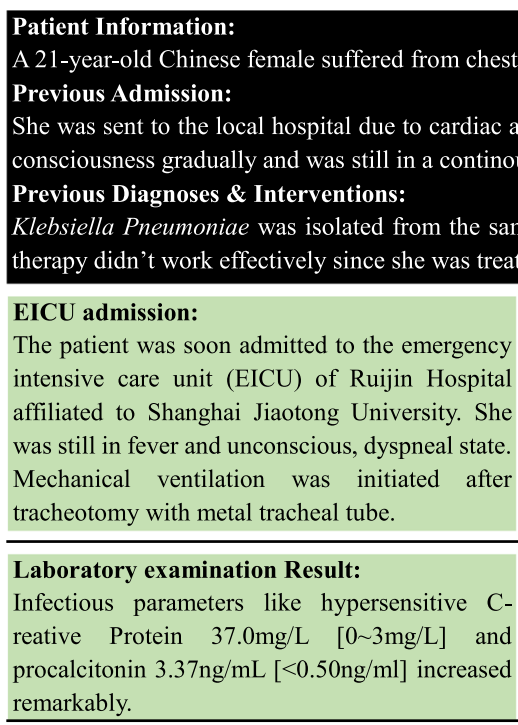

Patient Situation in 5.5 to 5.18:

Body temperature dropped to normal range stably. Repeated cultures of blood, sputum, vaginal secretion, and hip joint drainage fluid were made. MDR-KP were got in all samples which was susceptible to tigecycline, SMZ and PMB.

\section{Patient Situation on 5.21:}

The patient suffered from diarrhea suddenly with IAP increasing to $19 \mathrm{~cm} \mathrm{H}_{2} \mathrm{O}$. About $500 \mathrm{ml}$ black stool was found.

PMB-iSH occurrence:
Multiple diffused red rash shown up on the whole
body skin. With the rash fading away, SH with
brunet round spots was observed without pain and
pruritus. More dark brown spots was diffused on
the surface of trunk and limbs skin.

Additional Treatment:
PMB 500000 unit iv.gtt q12h

Laboratory examination Result:

Blood serum creatinine elevated to $430 \mu \mathrm{mol} / \mathrm{L}$ [53 97 $\mu \mathrm{mol} / \mathrm{L}]$. Significant reducing hemoglobin, $57 \mathrm{~g} / \mathrm{L}[113 \sim 151 \mathrm{~g} / \mathrm{L}]$ and distinctly decreasing platelet counts, $63 \times 10^{9} / \mathrm{L}\left[101 \sim 320 \times 10^{9} / \mathrm{L}\right]$.

\section{Last Outcome:}

At the urge of patients' relatives, the patient was transferred back to the local hospital for further treatment. Skin color didn't become shallow, not to mention recover as preivous state at that moment. In consideration of relatives will, we could not make a follow-up visit for the patients to investigate this event at last.

Fig. 5 Treatment timeline of this patient

Scale, PMB was probably associated with $\mathrm{SH}(+1$ for conclusive reports on this reaction, +2 for adverse events occurring after PMB administration, +1 for confirmed by objective evidences, +2 for the absence of possible alternative causes $=6$ points) $[13,14]$.

Besides PMB, the patient received omeprazole, meropenem, SMZ, colistin, tigecycline and heparin during her treatment period. Among these drugs, tigecycline and omeprazole could cause DiSH. It was reported that tigecycline could induce $\mathrm{SH}$, similar to minocycline [15]. However, SH occurred after the use of PMB for a few days, and their temporal correlation was found in this case. Hence, we considered PMB was the trigger of SH rather than tigecycline and omeprazole. In addition, drug-drug interaction should not be ignored. The combination of colistin and PMB may increase the risk of 
nephrotoxicity, which may cause the cumulation of PMB in vivo. Due to the limited medical resources and the critical condition of this patient, no further treatment was carried out for this issue.

However, it must be pointed out that acute renal insufficiency may be an important factor for PMB-iSH. Since up to $60 \%$ of PMB is excreted unchanged with urine and strongly associated with nephrotoxicity, its elimination half-life increases with reduced renal function. For individuals with renal impairment, reduced dose of PMB is required because of lower clearance rate. Back to this patient, her glomerular filtration rate (GFR) was almost $14.44 \mathrm{~mL} / \mathrm{min}$, indicating she suffered from severe acute kidney injury (AKI) during her treatment period. This could give rise to PMB accumulation so that the blood concentration would significantly increase. Although there is no direct evidence to prove that $\mathrm{PMB}-\mathrm{iSH}$ is a dose-dependent ADR, higher drug level in the body can be a related factor for skin darkening in some studies $[9,10]$.

The pathological mechanism of PMBiSH is still unclear. Overall, there are 4 possible basic pathological mechanisms of DiSH: (1) the most common drug-induced mechanism is the production and accumulation of melanin within cutaneous cells, especially in dermal macrophages; (2) the druge accumulation, associated with no melanin, usually exists within extracellular matrix or within dermal macrophages; (3) a specific drug plays a key role in the synthesis of other special pigments like lipofuscin; (4) drug-associated injury to dermal vessels usually results in the leakage of many red blood cells and the deposition of iron [1]. Most of relevant researches maintain that $\mathrm{DiSH}$ is attributed to an extensive inflammation, induced by inflammatory factors and producing allergic or toxic effects.

One probable mechanism of PMB-iSH is that PMB induces the release of histamine and the synthesis of melanin [11, 16, 17]. Histamine is released by basophils, mast cells and neurons in skin tissues, which can activate the inflammatory reaction by acting on 4 receptors $\left(\mathrm{H}_{1}\right.$ to $\left.\mathrm{H}_{4}\right)$ [18]. Yoshida M. et al. described the melanogenic effect of histamine: it could activate the $\mathrm{H}_{2}$ receptor of melanocytes, then up-regulate the activities of both tyrosinase and protein kinase A with the latter acting as a key role in melanogenesis [19]. Furthermore, it is proved that pigmented skin is more common in the head and neck since there are more melanocytes distributed in them [12].

Another presumed mechanism is related to epidermal langerhans cells. The research by Miori L. et al. revealed that the occurrence of $\mathrm{SH}$ meant the patient was at the stage of the post-inflammatory pathological progress, and the langerhans cells played a crucial role in chronic inflammatory skin diseases [20]. According to the histopathological results of a PMB-iSH patient's skin biopsy, Mattos K. P. H. et al. found that the greater proliferation of epidermal langerhans cells and dermal dendritic cells occurred indeed [12]. Based on the information above, it is presumed that PMB is an initiating factor of the pathological progress.

Last but not least, it was described by Choi $\mathrm{H}$. et al. that IL-6 could inhibit the proliferation of melanocytes and the generation of melanin [21]. Mattos K. P. H et al. found the expression of IL-6 was lower in several patients who suffered from the pigmented skin with $\mathrm{PMB}$ [12]. These research results may link the level of IL-6 to PMB-iSH. Further investigations should be carried out for verification.

The treatment of DiSH has already been challenging, let alone that of PMB-iSH. No specific treatment has been reported in literature up to now. This may be attributed to the fact that the illness is chronic and continuous progression that can easily relapse. For the patients whose symptoms can be relieved by lowering dosage, it is of great importance to find the balance between DiSH and therapeutic effects. What's more, it is necessary to find suitable alternative drugs and adjust the dosage in accordance with the patient' $\mathrm{s}$ physiological conditions and other clinical parameters. Another intervention is to avoid the exposure to sunlight, particularly ultraviolet ray, as much as possible so as to reduce the synthesis and accumulation of melanin. Topical or laser therapy through skin whitening agent may also be alternative ways to help the patients whose melanin concentrate within epidermal cells get rid of DiSH $[1,2]$.

Nowadays, it is still controversial on whether ceasing PMB can reverse the pathological change to pigmented skin and restore the original color. Zavascki A. P. et al. treated a 55-year-old male patient with hospital acquired pneumonia (HAP) by 14-day intravenous PMB treatment. His skin of the head and neck was evidently darkened during the treatment period. His skin tone was not restored to normal 3 months after PMB was withdrawn. On the basis of the Naranjo Adverse Drug Reaction probability scale, PMB was probably associated with hyperpigmentation (+2 for adverse events occurring after drug administration, +1 for confirmed by objective evidences, +2 for the absence of possible alternative cause $=5$ points) [22]. A 14-year-old female patient with aplastic anaemia who experienced an allogenic haematopoietic stem cell transplantation also suffered from PMB-iSH. On the 5th day of PMB treatment to treat postoperative infection, there was diffused $\mathrm{SH}$ and dark round spots on her upper body. Skin biopsy displayed that the patient underwent interface dermatitis with vacuolar damage. There were lots of melanophages and melanin pariticles within epidermal and dermal cells. Unlike the former patient, this patient was found with 
gradual recovery at the 3-month follow-up visit. Her skin color was restored to normal and the dark spots got shallow. And the spots almost disappeared at the 6-month follow-up visit [23].

There are some novel findings from our case. First of all, for the first time, it is observed that the appearance of red rashes is prior to the occurrence of PMB-iSH. We may infer that $\mathrm{PMB}-\mathrm{iSH}$ is possibly related to drug-induced hypersensitivity reaction, which is caused by the release of histamine and characterized as a symptom of rash and erythra. Secondly, based on our literature review, PMB-iSH mostly occurs on the skin of the head, neck and upper body. But in our case, not only did $\mathrm{SH}$ occur in the aforesaid locations, but also more serious symptoms were seen on the skin of the lower body and limbs.

In conclusion, $\mathrm{PMB}$ can give rise to $\mathrm{SH}$ indeed. Both clinicians and pharmacists should attach great importance to it. With the pathological mechanism still unclear, one of its probable reasons is associated with the release of histamine. Once PMB-iSH was observed, we should weigh the advantages and disadvantages of using PMB, and keep balance between curative effects and adverse event by such measures as dosage adjustment, cessation of drug administration and change to alternative antibiotics. Additionally, topical therapy and laser therapy can be treatments for PMB-iSH. Overall, further investigation is warranted.

\begin{abstract}
Abbreviations
ADR: Adverse drug reaction; AKI: Acute kidney failure; CPR: Cardiopulmonary resuscitation; CT: Computed tomography; DiSH: Drug-induced skin hyperpigmentation; EICU: Emergency intensive care unit; GIB: Gastrointestinal bleeding; HAP: Hospital acquired pneumonia; IAP: Intra-abdominal pressure; IL-6: Interleukin-6; MDR: Multi-drug resistance; MDR-KP: Multi-drug resistant Klebsiella pneumoniae; PMB: Polymyxin B; PMB-iSH: Polymyxin B-induced skin hyperpigmentation; SH: Skin hyperpigmentaion; SMZ: Sulfamethoxazole
\end{abstract}

\section{Acknowledgements}

The authors thank the staff of the EICU Department of Ruijin Hospital affiliated to Shanghai JiaoTong University School of Medicine for their facilities and collaboration.

\section{Funding}

This study was supported by Program for Outstanding Medical Academic Leader of Shanghai. This study was also funded by Shanghai Shen Kang Hospital Development Center Clinical Science and technology innovation project (No.SHDC12017116) and Important and weak discipline construction plan for health and family planning system of Shanghai (No.2016ZB0206), the biological medicine research program of Shanghai Municipal Science and Technology Commission (No.12411950500), and the National Natural Science Foundation of China (No. 81772107 and 81571931)

\section{Authors' contributions}

GHZ and JH conceived and designed this study. GHZ, LC, and ZQC performed the study. ZQC and EQM made substantial contributions to acquisition of data, and involved in drafting the manuscript. GHZ and LC wrote the paper. ZQC and EQM made substantial contributions to general supervision of the research group. EZC made substantial contributions to acquisition, analysis and interpretation of the data, supported the present study with national fundings, and provided the analysis tools. JH and EZC reviewed and revised the manuscript critically for important intellectual content, and approved the final version. All authors read and approved the manuscript.

\section{Ethics approval and consent to participate}

This study was approved by Ruijin Hospital Institutional Review Board and has been performed in accordance with the ethical standards laid down in "Declaration of Helsinki 1964" and its later amendments or comparable ethical standards. One patient was enrolled in the study and informed consent forms were signed by this patient.

\section{Consent for publication}

Written informed consent was obtained from the patient to the publication of this case report. A copy of the written informed consent is available for the review by the editor of this journal.

\section{Competing interests}

The authors declare that they have no competing interests.

\section{Publisher's Note}

Springer Nature remains neutral with regard to jurisdictional claims in published maps and institutional affiliations.

\section{Author details}

1Department of Pharmacy, Xiaolan Hospital Affiliated to Southern Medical University, Zhongshan, China. ${ }^{2}$ Department of Pharmacy, Zhongshan People's Hospital, Zhongshan, China. ${ }^{3}$ Department of Emergency Intensive Care Unit, Ruijin Hospital Affiliated to Shanghai JiaoTong University School of Medicine, Shanghai, China. ${ }^{4}$ Department of Emergency Intensive Care Unit, Ruijin Hospital Affiliated to Shanghai JiaoTong University School of Medicine, EICU 3 Floor, 5 Building, 197 Ruijin No. 2 Road, Huangpu District, Shanghai, China. ${ }^{5}$ Department of Pharmacy, Ruijin Hospital Affiliated to Shanghai JiaoTong University School of Medicine, 202 Room, 12 Building, 197 Ruijin No. 2 Road, Huangpu District, Shanghai, China.

Received: 27 February 2018 Accepted: 20 June 2018

Published online: 04 July 2018

\section{References}

1. Dereure O. Drug-induced skin pigmentation. Epidemiology, diagnosis and treatment. Am J Clin Dermatol. 2001;2(4):253.

2. Krause W. Drug-induced hperpigemntation: a systematic review. J Dtsch Dermatol Ges. 2013;11(7):644-51.

3. Nation RL, Li J, Cars O, Couet W, Dudley MN, Kaye KS, Mouton JW, Paterson $\mathrm{DL}$, Tam VH, Theuretzbacher U. Framework for optimisation of the clinical use of colistin and polymyxin B: the Prato polymyxin consensus. Lancet Infect Dis. 2015:15(2):225.

4. Tran TB, Velkov T, Nation RL, Forrest A, Tsuji BT, Bergen PJ, Li J. Pharmacokinetics/pharmacodynamics of colistin and polymyxin B: are we there yet? Int J Antimicrob Agents. 2016;48(6):592-7.

5. Velkov T, Dai C, Ciccotosto GD, Cappai R, Hoyer D, Li J. Polymyxins for CNS infections: pharmacology and neurotoxicity. Pharmacol Ther. 2017;

6. Kelesidis T, Falagas ME. The safety of polymyxin antibiotics. Expert Opin Drug Saf. 2015;14(11):1687-701

7. Knueppel RC, Rahimian J. Diffuse cutaneous hyperpigmentation due to tigecycline or polymyxin B. Clin Infect Dis. 2007;45(1):136

8. Lahiry S, Choudhury S, Mukherjee A, Bhunya PK, Bala M. Polymyxin Binduced diffuse cutaneous hyperpigmentation. J Clin Diagn Res. 2017;11(2): FD01-2.

9. Gothwal S, Meena K, Sharma SD, Polymyxin B. Induced generalized hyperpigmentation in neonates. Indian J Pediatr. 2016;83(2):179-80.

10. Shih LK, Gaik CL. Polymyxin B induced generalized skin hyperpigmentation in infants. J Pediatr Scis. 2014;6:e215.

11. Mattos KP, Lloret GR, Cintra ML, Gouvea IR, Betoni TR, Mazzola PG, Moriel P. Acquired skin hyperpigmentation following intravenous polymyxin B treatment: a cohort study. Pigment Cell Melanoma Res. 2016;29(3):388-90.

12. Mattos KPH, Cintra ML, Gouvea IR, Ferreira LA, Velho P, Moriel P. Skin hyperpigmentation following intravenous polymyxin $B$ treatment associated with melanocyte activation and inflammatory process. J Clin Pharm Ther. 2017;42(5):573-8.

13. Naranjo CA, FRCPC DNHSM, Lanctôt MKL. Advances in the diagnosis of adverse drug reactions. J Clin Pharmacol. 1992;32(10):897-904. 
14. Naranjo CA, Busto U, Sellers EM, Sandor P, Ruiz I, Roberts EA, Janecek E, Domecq C, Greenblatt DJ. A method for estimating the probability of adverse drug reactions. Clin Pharmacol Ther. 1981;30(2):239.

15. Vandecasteele SJ, De Ceulaer J, Wittouck E. Tigecycline induced hyperpigmentation of the skin. Open Forum Infect Dis. 2016;3(1):ofw033.

16. Fechner GA, Jacobs JJ, Parsons PG. Inhibition of melanogenesis in human melanoma cells by novel analogues of the partial histamine $(\mathrm{H} 2)$ agonist nordimaprit. Biochem Pharmacol. 1993;46(1):47.

17. Voitenko VG, Bayramashvili DI, Zebrev Al, Zinchenko AA. Relationship between structure and histamine releasing action of polymyxin $B$ and its analogues. Agents Actions. 1990;30(1-2):153.

18. Marone G, Granata F, Spadaro G, Genovese A, Triggiani M. The histaminecytokine network in allergic inflammation. J Allergy Clin Immunol. 2003; 112(4 Suppl):83-8.

19. Yoshida M, Takahashi Y, Inoue S. Histamine induces Melanogenesis and morphologic changes by protein kinase a activation via $\mathrm{H} 2$ receptors in human normal melanocytes. J Investig Dermatol. 2000;114(2):334-42.

20. Miori L, Vignini M, Rabbiosi G. Flagellate dermatitis after bleomycin. A histological and immunohistochemical study. Am J Dermatopathol. 1990; 12(6):598-602.

21. Choi H, Kim K, Han J, Choi H, Jin SH, Lee EK, Shin DW, Lee TR, Lee AY, Noh M. Kojic acid-induced IL-6 production in human keratinocytes plays a role in its anti-melanogenic activity in skin. J Dermatol Sci. 2012;66(3):207-15.

22. Zavascki AP, Manfro RC, Maciel RA, Falci DR. Head and neck hyperpigmentation probably associated with Polymyxin B therapy. Ann Pharmacother. 2015;49(10):1171-2.

23. Zavascki AP, Schuster LF, Duquia RP. Histopathological findings of pigmented lesion and recovery of natural skin colour in a patient with polymyxin B-associated diffuse hyperpigmentation. Int J Antimicrob Agents. 2016:48(5):579-80

\section{Ready to submit your research? Choose BMC and benefit from}

- fast, convenient online submission

- thorough peer review by experienced researchers in your field

- rapid publication on acceptance

- support for research data, including large and complex data types

- gold Open Access which fosters wider collaboration and increased citations - maximum visibility for your research: over $100 \mathrm{M}$ website views per year 